\title{
LA SEGURIDAD EN LA POLITICA EXTERIOR DE PEÑA NIETO: EL INVITADO INCÓMODO
}

\author{
SECURITY IN THE FOREIGN POLICY OF PEÑA NIETO: \\ THE UNINVITED GUEST
}

\section{LA SÉCURITÉ ET SA PLACE DANS LA POLITIQUE ÉTRANGÈRE DE PEÑA NIETO: UN INVITÉ GÊNANT}

\author{
Jorge Chabat \\ Universidad de Guadalajara \\ jorgechabat@gmail.com
}

Resumen: El artículo analiza la política exterior del presidente Enrique Peña Nieto en el aspecto de seguridad. Se hace una revisión de los planteamientos en política exterior de su administración y su intento por "recuperar" el liderazgo de México como "potencia emergente", así como el énfasis en las reformas económicas y la intención de "deseguritizar" la política exterior. El artículo plantea que hubo tres factores que hicieron que al final fracasara el propósito de deseguritización de la política exterior y que, en cambio, se colara como un invitado incómodo: el repunte en la tasa de homicidios a partir de 2014, las crisis provocadas por la desaparición de los estudiantes de la normal de Ayotzinapa y el asesinato de varios presuntos criminales en Tlatlaya por las fuerzas armadas, así como la llegada a la presidencia de Estados Unidos de Donald Trump, que hizo que la seguridad se convirtiera en una carta importante de negociación frente a ese país. El texto concluye que, aun si los procesos de seguritización o deseguritización son actos retóricos de los actores estatales, al final deben tener una correspondencia con la realidad.

Palabras clave: Enrique Peña Nieto; política exterior; seguridad; seguritización; Ayotzinapa; Donald Trump.

Abstract: This paper analyzes the foreign policy of President Enrique Peña Nieto in terms of security. It first reviews the foreign policy approaches of his administration and his attempt to "restore" Mexico's leadership as an "emerging power," as well as the emphasis on economic reforms and the intention to "desecuritize" foreign policy. The article argues that there were three factors 
that ultimately caused the intention of desecuritizing foreign policy to fail and that, instead, led it to become an uninvited guest: the surge in the homicide rate from 2014, the crisis arising from the disappearance of students from the Ayotzinapa normal college, and the murder of a number of alleged criminals in Tlatlaya by the armed forces, as well as the arrival of Donald Trump to the presidency of the United States, which made security an important card to play in negotiating with that country. The text concludes that, even if the processes of securitization or desecuritization are rhetorical tropes used by state actors, in the end they must correspond with reality.

Keywords: Enrique Peña Nieto; foreign policy; security; securitization;

Ayotzinapa; Donald Trump.

\section{Traducción de Gonzalo Celorio Morayta}

RésumÉ: L'article analyse la politique étrangère du président Enrique Peña Nieto en ce qui concerne la sécurité. Il souligne les thèses de cette politique, les efforts du gouvernement dans le but de "récupérer" un rôle de leader pour le Mexique en tant que "puissance émergente", l'insistance sur les réformes économiques et l'intention de "désécuriser" la politique étrangère. On a raté ce dernier objectif -et la question de la sécurité s'est imposée alors comme un invité gênant- pour trois raisons: l'élévation du taux d'homicides; deux crises, dont l'une a découlé de la disparition d'étudiants de l'école normale d'Ayotzinapa, l'autre du meurtre de criminels présumés aux mains des militaires à Tlatlaya; et l'arrivée de Donald Trump à la présidence des ÉtatsUnis, après laquelle la sécurité est devenue une carte de négociation importante dans les rapports de cette puissance avec le Mexique. La conclusion de l'article est que, même si les processus de "sécurisation" ou de "désécurisation” font partie de la rhétorique des États, il faut bien qu'ils correspondent à des faits réels.

Mots clefs: Enrique Peña Nieto; politique étrangère; sécurité; "sécurisation"; Ayotzinapa; Donald Trump.

Traducción de BERNARdo Mabire

Fecha de recepción: diciembre de 2018

Fecha de aceptación: febrero de 2019 
$\mathrm{L}$ A victoria de Enrique Peña Nieto en las elecciones de 2012 significó el regreso del Partido Revolucionario Institucional (PRI) a la Presidencia de México, después de 12 años y luego de que fuera derrotado en 2000 por el candidato del Partido de Acción Nacional (PAN), Vicente Fox. Si bien el triunfo de Peña Nieto no fue como los que solían disfrutar los candidatos del PRI en las épocas doradas de su hegemonía, con porcentajes de votos mayores al $50 \%$, la distancia respecto al segundo lugar (6.6\% de diferencia) le dio la suficiente legitimidad para plantear cambios radicales frente a los gobiernos anteriores. En ese sentido, uno de los temas centrales de su campaña fue modificar la estrategia de seguridad de su antecesor, Felipe Calderón, a la cual una parte de la opinión pública le atribuyó la responsabilidad por el incremento en la tasa de homicidios a partir de 2007. De esta forma, no es de extrañar que, desde el inicio de su gobierno, Peña Nieto se haya planteado "deseguritizar" la política interna y externa como una manera de marcar una clara distancia frente al sexenio anterior. Al mismo tiempo, su política exterior buscó retomar los postulados desarrollados por el PRI en la segunda mitad del siglo pasado, como una forma de marcar el regreso de lo que la propia propaganda gubernamental de esos años definió como una era dorada en los asuntos internacionales. No obstante, este empeño no fue exitoso pues, como veremos en el presente trabajo, a pesar de los esfuerzos por restar importancia al tema de la seguridad dentro y fuera de México, al final la realidad acabó por rebasar a la retórica gubernamental y la globalización en la que está inmerso el país hizo imposible regresar a la política exterior del siglo pasado. En la primera parte de este artículo

${ }^{1}$ El término "seguritización" fue acuñado por Barry Buzan, Ole Waever y Jaap de Wilde para referirse al proceso por el que los actores estatales transforman algunos temas en asuntos de seguridad, al darles una connotación de amenaza, con lo cual éstos obtienen una categoría de urgencia que les permite atraer recursos extraordinarios y no estar sujetos a las reglas establecidas. Barry Buzan, Ole Wæver y Jaap de Wilde, Security: A New Framework for Analysis, Boulder, Lynne Rienner Publishers, 1998. 
analizaremos el contexto en el que llega Peña Nieto a la Presidencia, así como sus planteamientos iniciales en campaña. En una segunda parte veremos la forma en que se desarrolló la política exterior respecto al tema de seguridad y el interés del gobierno en reducir la visibilidad de éste. Posteriormente analizaremos las crisis de seguridad que deterioraron la imagen internacional del país y la forma en que se condujo el tema en la relación con Estados Unidos, así como el fracaso en el intento por deseguritizar la política exterior. Finalmente, plantearemos algunas conclusiones.

La hipótesis de este artículo es que el proceso de deseguritización de la política externa de Peña Nieto fracasó por tres factores: el repunte en la tasa de homicidios a partir de 2014, varios acontecimientos puntuales que dañaron la imagen internacional de México en el tema de la seguridad -como la desaparición de los estudiantes de la normal de Ayotzinapa-y la llegada al poder de Donald Trump, que hizo del tema de la seguridad, precisamente, una de las principales armas de negociación del gobierno de México.

\section{El contexto de la victoria de Peña Nieto}

El gobierno de Felipe Calderón, quien precedió a Peña Nieto en la Presidencia, tuvo como una de sus principales prioridades el combate a la delincuencia organizada, en particular, el narcotráfico. Dicha prioridad permeó su política interna y externa. Mucho se ha especulado sobre las razones de ello y sobre si esta decisión presidencial fue la causante del incremento en la tasa de homicidios durante su sexenio. Sin embargo, más allá de esa discusión, lo cierto es que una parte de la opinión pública aceptó la versión sostenida por los opositores a Calderón de que la responsable directa del incremento de la violencia en el país fue la estrategia seguida por su gobierno. Esta versión fue sostenida tanto por la izquierda, agrupada en torno al Partido de la Revolución Democrática y su entonces candidato, Andrés Manuel López Obrador, 
como por el PRI, a pesar de que al final del sexenio de Calderón la tasa de homicidios comenzó a registrar una baja significativa. En este sentido, no resulta extraño que el candidato priista a la Presidencia, Enrique Peña Nieto, hiciera de la crítica a la estrategia de seguridad de Calderón uno de sus temas de campaña. En abril de 2012, opinó negativamente sobre la estrategia gubernamental para combatir al crimen organizado por ser "notoriamente fallida, como lo evidencia su altísimo y doloroso costo en vidas humanas". ${ }^{2}$ Asimismo, Peña Nieto afirmó: "en mi gobierno vamos a reformular la estrategia a partir del paradigma de seguridad democrática y ciudadana, que propone atender los orígenes de la violencia y ofrecer alternativas a la vida criminal". ${ }^{3}$ En esta misma línea, el candidato priista dijo que si bien la decisión "de enfrentar con determinación a la delincuencia organizada es correcta", se partió de "una comprensión limitada del problema" y se buscó "una solución concentrada únicamente en el uso de la fuerza". ${ }^{4}$ De forma paralela a esta crítica a la estrategia de seguridad de Calderón, Peña Nieto planteó desde su campaña la importancia de generar crecimiento económico, ${ }^{5}$ tema que se convirtió en el eje de su gobierno al proponer varias reformas estructurales, como la energética, la hacendaria y la laboral. ${ }^{6}$

2 "Peña propone reformular estrategia de seguridad", El Economista, 28 de abril de 2012, https://www.eleconomista.com.mx/politica/Penapropone-reformular-estrategia-en-seguridad-20120428-0004.html (consulta del 17 de diciembre de 2018).

${ }^{3}$ Loc. cit.

${ }^{4}$ Loc. cit.

5 "Peña Nieto presenta propuestas de gobierno en Davos", El Economista, 27 de enero de 2012, https:/ /www.eleconomista.com.mx/politica/ Pena-Nieto-presenta-propuestas-de-gobierno-en-Davos-20120127-0012. html (consulta del 17 de diciembre de 2018).

6 "Peña Nieto prepara paquete de reformas estructurales", El Economista, 2 de julio de 2012, https:/ /www.eleconomista.com.mx/economia/ Pena-Nieto-prepara-paquete-de-reformas-estructurales-20120702-0102. html (consulta del 17 de diciembre de 2018). 
En la búsqueda de votos, anunció dos semanas antes de la elección que el general Óscar Naranjo, quien fuera director de la Policía Nacional de Colombia, sería su asesor externo en materia de seguridad. Incluso, el propio Naranjo dijo unos días después de la elección que la meta del gobierno entrante era que la violencia bajara $50 \%$ durante los primeros cien días. ${ }^{7}$ Asimismo, como parte de sus promesas de campaña, Peña Nieto propuso en mayo de 2012 crear un cuerpo nacional de gendarmería con elementos de las fuerzas armadas para reforzar los cuerpos policiacos en el combate a la delincuencia. ${ }^{8}$

Como puede apreciarse, uno de los elementos centrales de la plataforma de Peña Nieto en su campaña por la Presidencia fue el planteamiento de una estrategia de seguridad claramente diferenciada de la seguida por Calderón, con menor énfasis en el uso de la fuerza. Otro elemento fundamental fue la importancia del crecimiento económico y las reformas estructurales. En este sentido, era evidente la intención de deseguritizar la política de su administración, así como darle un mayor contenido económico. Estas prioridades se reflejaron también en su política exterior.

\section{La POlítica eXterior de Peña Nieto}

Desde su campaña, Peña Nieto delineó lo que serían sus prioridades de política exterior. El propósito era "recuperar" el liderazgo de México como "potencia emergente". ${ }^{9}$ El supuesto que estaba detrás de estas prioridades era que, ante los cambios globales, México se había "desdibujado" y compara-

7 "Naranjo propone plan para reducir violencia en México en 100 días”, El Espectador, 7 de julio, https://www.elespectador.com/noticias/elmundo/naranjo-propone-plan-reducir-violencia-mexico-100-dias-articu lo-357979 (consulta del 17 de diciembre de 2018).

${ }^{8}$ Claudia Guerrero, "Plantea Peña Nieto crear Gendarmería", Reforma, Ciudad de México, 10 de mayo de 2012.

${ }^{9}$ Enrique Peña Nieto, México, la gran esperanza, México, Grijalbo, 2012. 
ba la situación del país con "el despegue de Brasil". El trasfondo de esta política era la idea de que, en el pasado, México había tenido un papel protagónico en "la intermediación y orquestación de iniciativas regionales y multilaterales para resolver situaciones delicadas en tiempos de crisis", como el Tratado de Tlatelolco, el Grupo Contadora, la primera Cumbre Iberoamericana o la firma del Tratado de Libre Comercio de América del Norte. Para que México pudiera ser un actor global, Peña Nieto planteaba siete acciones prioritarias: 1) impulsar una mejor integración de América del Norte, 2) fortalecer la cooperación con Centroamérica y el Caribe, 3) recuperar la interlocución efectiva con América del Sur, 4) consolidarnos como potencia cultural iberoamericana, 5) consolidar una alianza con la región de Asia-Pacífico, 6) impulsar un multilateralismo efectivo y 7) dinamizar la relación con la Unión Europea. Para Peña Nieto era necesario el fortalecimiento interno, a través del ejercicio real de derechos fundamentales y de altas tasas de crecimiento, así como una "estrategia de alianzas" guiada por estas prioridades. El propósito final era "traer más oportunidades y mejores condiciones de vida para los mexicanos e incidir en las decisiones que definen el rumbo de las relaciones internacionales". En otras palabras, la política exterior buscaba, como en el sexenio de Carlos Salinas de Gortari, ser una palanca para el desarrollo económico, ${ }^{10}$ meta que se convertiría en un mantra de su accionar internacional.

Después de haber asumido la Presidencia en diciembre de 2012, Peña Nieto definió con más precisión su proyecto de política internacional. En una reunión con miembros del Servicio Exterior Mexicano, estableció los "cuatro pilares"

${ }^{10}$ Algunos analistas señalaron que desde la administración de Salinas de Gortari la política exterior se había "economizado". Jorge Chabat, "Mexico's foreign policy in 1990: Electoral Sovereignty and integration with the United States", Journal of Interamerican Studies and World Affairs, vol. 33, núm. 4 (1991), pp. 1-25; Jorge G. Castañeda, "Salinas' international relations gamble", Journal of International Affairs, Vol. 43, núm. 2 (1990), pp. 407-422. 
de su política en esta materia: ${ }^{11}$ 1) fortalecer la presencia de México en el exterior, con el propósito de "estrechar nuestras relaciones bilaterales y ampliar nuestra capacidad para incidir en la conformación de un orden internacional más próspero y equitativo"; 2) ampliar la cooperación internacional a fin de contribuir al "logro de los objetivos de desarrollo interior"; 3) promover el valor de México en el mundo, para que se conozca "nuestra cultura, nuestra historia, nuestras artes, nuestras bellezas naturales y especialmente la oportunidad económica y el buen momento que México hoy está viviendo", y 4) velar por "los asuntos e intereses de México y los mexicanos en el exterior". Esta definición confirmó que el eje de la nueva política exterior iba a ser la atracción de recursos económicos para promover el desarrollo del país, lo cual se reflejó en el nombramiento como secretario de Relaciones Exteriores de José Antonio Meade quien, además de poseer un doctorado en Economía por la Universidad de Yale, había sido secretario de Hacienda y Crédito Público en el gobierno de Calderón, de 2010 a 2012, cargo que volvería a ocupar más tarde en el gobierno de Peña Nieto, de septiembre de 2016 a noviembre de 2017.

La prioridad económica fue señalada expresamente por el canciller Meade en abril de 2013: México debía alcanzar el crecimiento y la participación en el comercio internacional a través de la política exterior. ${ }^{12}$ Así, la "economización" de la política exterior se reflejó también en el interés del gobierno de Peña Nieto en el Tratado de Asociación Transpacífico (TPP por sus siglas en inglés) durante la reunión en Indonesia del Foro de Cooperación Económica Asia-Pacífico (APEC,

11 "México se compromete a ser un actor con mayor responsabilidad global: Enrique Peña Nieto”, México, Presidencia de la República, 14 de febrero de 2013, http://www.presidencia.gob.mx/articulos-prensa/mexi co-se-compromete-a-ser-un-actor-con-mayor-responsabilidad-global-enri que-pena-nieto/ (consulta del 14 de octubre de 2014).

12 Eduardo Camacho, "sRE: política exterior apoyará al comercio”, El Universal, 17 de abril de 2013, http://www.eluniversal.com.mx/finan zas/101867.html (consulta del 17 de diciembre de 2018). 
por sus siglas en inglés), en octubre de 2013, ocasión en la cual se reunió con los mandatarios asistentes. ${ }^{13}$ En el mismo sentido, procuró un acercamiento con la República Popular China. ${ }^{14}$

Detrás de los planteamientos iniciales en política exterior estaba la convicción de que México debía desempeñar un papel protagónico en la arena mundial basado en su potencial económico. En esta imagen no cabía la idea de un país acosado por la inseguridad y a la defensiva. De acuerdo con el propio Peña Nieto, éste sería el "momento de México", ${ }^{15}$ en el que el país recuperaría el liderazgo internacional del pasado.

\section{UN "MÉXico EN PAZ"}

El énfasis en el desarrollo económico se compaginaba muy bien con el intento de deseguritizar la agenda gubernamental. Por ello, en el Plan Nacional de Desarrollo 2013-2018 se estableció como uno de los objetivos el lograr un "México en paz”, meta que también formó parte de la política exterior ${ }^{16}$ $y$, por cierto, empataba muy bien con una de las característi-

${ }^{13}$ Erika Hernández, "Peña Nieto asegura que afianzó lazos con Asia", Terra Noticias, 8 de octubre de 2012, http://noticias.terra.com.mx/mexi $\mathrm{co} /$ politica/pena-nieto-asegura-que-mexico-afianzo-lazos-con-asia,66ab 808345891410VgnVCM5000009ccceb0aRCRD.html (consulta del 14 de octubre de 2014).

${ }^{14}$ Kenia Ramírez Meda y Nalia María Rochin Aguilar, "La política exterior de México durante el sexenio de Enrique Peña Nieto", Journal of International Relations, núm. 8 (2017), pp. 51-66.

${ }^{15}$ Enrique Peña Nieto, "Mexico's moment", The Economist, 21 de noviembre de 2012, https://www.economist.com/news/21566314-enrique pe\% C3\%B1a-nieto-mexicos-newly-elected-president-sets-out-his-priori ties-mexicos-moment (consulta del 17 de diciembre de 2018).

16 Tania L. Montalvo, "Un México en paz debe tener el apoyo de la diplomacia: canciller Meade”, Expansión, 7 de enero de 2013, https:// expansion.mx/nacional/2013/01/07/un-mexico-en-paz-debe-tener-elapoyo-de-la-diplomacia-canciller-meade (consulta del 17 de diciembre de 2018). 
cas de la "época dorada" de la diplomacia mexicana de los años sesenta y setenta del siglo pasado: el "pacifismo". En esa misma línea, el gobierno mexicano anunció varias medidas que buscaban redefinir el tema de la seguridad tanto interna como externamente. Una primera decisión, que buscaba marcar una clara diferencia con la política seguida por el gobierno de Calderón, fue la desaparición de la Secretaría de Seguridad Pública, dependencia que había sido identificada por la oposición como la encargada de instrumentar una estrategia de seguridad que, desde esa óptica, era la causa del aumento en la tasa de homicidios. Ello implicó que la rectoría del Sistema Nacional de Seguridad Pública y las funciones de la Policía Federal fueran absorbidas por la Secretaría de Gobernación, la cual se encargaría, además, de desarrollar un programa de prevención del delito, en la línea marcada por Peña Nieto de usar menos la fuerza y atender más las causas sociales. El Programa Nacional para la Prevención Social de la Violencia y el Delito (PNPSVD) se proponía "atender los factores de riesgo y de protección vinculados a la violencia y la delincuencia” y tenía cinco objetivos:

1. Incrementar la corresponsabilidad de la ciudadanía y de los actores sociales en la prevención social mediante su participación y desarrollo de competencias.

2. Reducir la vulnerabilidad ante la violencia y la delincuencia de las poblaciones de atención prioritaria.

3. Generar entornos que favorecieran la convivencia y seguridad ciudadana.

4. Fortalecer las capacidades institucionales para la seguridad ciudadana en los gobiernos municipales/delegacionales, estatales y federal.

5. Asegurar la coordinación entre las dependencias y entidades de la administración pública federal para la implementación de programas de prevención social. ${ }^{17}$

17 “¿Qué es el Programa Nacional para la Prevención social de la Violencia y el Delito?”, http://mexicoevalua.org/prevencion/conoce-el- 
Por otro lado, en agosto de 2014, se publicó el decreto para crear la División de Gendarmería como parte de la Policía Federal. ${ }^{18}$ Asimismo, en abril de 2013 se había anunciado que la Secretaría de Gobernación sería el único canal con el gobierno de Estados Unidos en temas de seguridad. ${ }^{19}$ Para complementar esta serie de reformas en la materia, en 2016 se anunció la creación del Centro de Nacional de Fusión de Inteligencia, que buscaba coordinar la recopilación de información sobre la inseguridad en el país a través de cinco centros regionales. ${ }^{20}$

\section{LA SEGURIDAD EN LA POLÍTICA EXTERIOR}

La “economización” de la política exterior, el énfasis en deseguritizar la agenda interna y la idea de regresar a la "época dorada" de la acción internacional de México provocaron que dicha política tuviera, en general, un bajo perfil. A pesar de ello, se mantuvieron algunos esquemas de cooperación en seguridad con Estados Unidos, principalmente la Iniciativa Mérida, aunque ésta dejó de ser un punto de referencia de la relación bilateral. Asimismo, el gobierno mexicano ins-

proyecto/que-es-el-programa-nacional-para-la-prevencion-social-de-laviolencia-y-la-delincuencia-pronapred/ (consulta del 17 de diciembre de 2018).

18 "Segob publica decreto que crea la División de Gendarmería”, El Financiero, 22 de agosto de 2014, http://www.elfinanciero.com.mx/socie $\mathrm{dad} /$ segob-publica-decreto-que-crean-la-division-de-gendarmeria (consulta del 17 de diciembre de 2018) .

19 "Gobernación, única ventanilla para tratar temas de seguridad con Estados Unidos”, Animal Político, 30 de abril de 2013, https: / / www.animalpolitico.com/2013/04/gobernacion-la-unica-ventanilla-para-tratar-temade-seguridad-con-eu/ (consulta del 17 de diciembre de 2018).

20 Susana Guzmán, "Centro de fusión de inteligencia coordinará acciones para fortalecer seguridad: Segob", El Financiero, 2 de noviembre de 2016, http:/ /www.elfinanciero.com.mx/nacional/centro-de-fusion-de-in teligencia-coordinara-acciones-para-fortalecer-seguridad-segob (consulta del 17 de diciembre de 2018). 
tituyó en 2016 el Grupo de Alto Nivel con Washington, el cual buscaba abordar los temas de la relación bilateral, en particular los aspectos de seguridad. ${ }^{21}$ Dicho grupo se asemejó al Grupo de Contacto de Alto Nivel que crearon los dos países en 1996 para atender las crisis en torno al tema del narcotráfico. En la relación con América Latina, el gobierno de Peña Nieto mantuvo diversos grupos de alto nivel en seguridad (Ganseg) con Guatemala, Panamá, Perú, El Salvador y Honduras, los cuales celebraron reuniones periódicas para reforzar la cooperación ante amenazas comunes.

Mención aparte merece la iniciativa de México, Colombia y Guatemala, que data de 2012, para la realización de la Sesión Especial de la Asamblea General de Naciones Unidas (UNGAss) sobre Drogas, la cual tuvo lugar en abril de 2016. La UNGAss sobre Drogas anterior, llevada a cabo en 1998, fue también promovida por México y en ella se buscó poner énfasis en el papel de la demanda y de los países consumidores en el problema de las drogas ilícitas. No obstante, en la de 2016 el enfoque fue mucho más crítico del régimen internacional de control de drogas y reflejó la fractura en el consenso en torno al mismo. ${ }^{22}$ Dicha reunión sirvió también como foro para que el gobierno mexicano mostrara un cambio en su tradicional posición de moderación sobre el régimen de drogas. En la reunión en Nueva York, Peña Nieto expresó una crítica abierta a este régimen: "El esquema basado esencialmente en el prohibicionismo, la llamada guerra contra las drogas, que inició en los años setenta no ha logrado inhibir la producción, el tráfico, ni el consumo de drogas en el mundo". Y añadió:

21 Natalia Gómez y Ariadna García, "México y EU forman grupo de alto nivel en seguridad", El Universal, 12 de octubre de 2016, http:/ / www. eluniversal.com.mx/articulo/nacion/seguridad/2016/10/12/mexico-yeu-forman-grupo-de-alto-nivel-en-seguridad (consulta del 17 de diciembre de 2018).

${ }^{22}$ Sobre la fractura en el consenso en torno al régimen internacional de control de drogas, véase David R. Bewley-Taylor, International Drug Control: Consensus fractured, Cambridge, University Press, 2012. 
Como pocos, conocemos las limitaciones y las dolorosas implicaciones del paradigma eminentemente prohibicionista. Por eso, durante mi administración, se ha buscado atender el fenómeno de las drogas de una forma más integral, con una estrategia que evita generar mayor violencia y en la que prácticamente participan todas las secretarías del gobierno de México. ${ }^{23}$

Dicha posición, que muy probablemente lleve a eliminar la prohibición del uso de la cannabis en el sexenio del presidente Andrés Manuel López Obrador, y a regularlo, compaginaba muy bien con la idea de adoptar un enfoque menos punitivo en el tema del narcotráfico.

En el ámbito multilateral habría que mencionar también la decisión del gobierno de Peña Nieto de destinar tropas a las fuerzas de mantenimiento de paz de la Organización de las Naciones Unidas, la cual podría parecer contradictoria con la política de un "México en paz", aunque más bien lo que buscaba era apoyar procesos de pacificación en otras partes del mundo, en correspondencia con la tradición pacifista de México.

\section{LA CRISIS DE SEGURIDAD Y EL REGRESO DEL TEMA A LA AGENDA DE LA POLÍTICA EXTERIOR}

Hasta 2014, todo indicaba que los intentos por deseguritizar la agenda interna y externa de Peña Nieto habían sido exitosos. De hecho, la percepción de la opinión pública así lo confirmaba. Según una encuesta dada a conocer en noviembre de $2013,56 \%$ de la población pensaba en junio de 2012, al final del sexenio de Calderón, que el presidente de la República había sido rebasado por el crimen organizado. Dicha

23 Naciones Unidas, "Boletín onu. Discurso del presidente Enrique Peña Nieto en la ungass 2016”, Comunicado núm. 16/083,19 de abril de 2016, http:/ /www.cinu.mx/comunicados/2016/04/el-problema-mundialde-las-dro/ (consulta del 17 de diciembre de 2018). 
percepción cambió radicalmente diez meses después, porque en abril de 2013, ya en el sexenio de Peña Nieto, $56 \%$ estimaba que el jefe del Ejecutivo tenía capacidad para mantener el control. Sin embargo, la opinión volvió a pendular en sólo seis meses, pues ya en octubre de ese año $60 \%$ pensaba, una vez más, que el presidente ya había sido rebasado por la delincuencia. ${ }^{24}$ Esta percepción de inseguridad coincidió con el deterioro de la situación en Michoacán, que llevó a la intervención del Ejército a principios de ese año y al nombramiento de un comisionado del gobierno federal a principios de 2014, quien se convirtió en el gobernador de facto de la entidad. Ya para entonces, la imagen internacional del gobierno empezaba a deteriorarse y el tema de la seguridad se colaba en la agenda de la política exterior. Las noticias sobre los grupos de autodefensa michoacanos eran cada vez más comunes en la prensa de otros países, la cual veía el surgimiento de estos grupos como el "ejemplo más notable de la debilidad policial [...] especialmente en las áreas rurales, donde la frustración y la falta de confianza en las instituciones es más profunda". ${ }^{25}$ Pero si bien la crisis en Michoacán afectó seriamente la imagen de que el gobierno tenía "bajo control" el problema de la inseguridad, lo cierto es que fue la desaparición de 43 estudiantes de la normal rural de Ayotzinapa, en septiembre de 2014, lo que derrumbó esa imagen y colocó la seguridad en lo alto de la agenda nacional e internacional.

A pesar de que la evidencia indica que la desaparición de los estudiantes fue resultado de la complicidad de las policías de Iguala y Cocula con un grupo local del crimen orga-

${ }^{24}$ Ulises Beltrán y Alejandro Cruz, "Crece percepción de violencia”, Excélsior, 11 de noviembre de 2013, http://www.bgc.com.mx/archivos/ excelsior/2013/noviembre/EX111113.pdf (consulta del 17 de diciembre de 2018).

25 Randall C. Archibold, "A Quandary for Mexico as vigilantes rise", The New York Times, 15 de enero de 2014, https://www.nytimes.com/ 2014/01/16/world/americas/a-quandary-for-mexico-as-vigilantes-rise. html (consulta del 17 de diciembre de 2018). 
nizado, la ineficacia en la investigación de la Procuraduría General de la República (PGR) transfirió pronto el costo político de tal suceso al gobierno federal. Evidentemente, los partidos de oposición buscaron obtener una ganancia política del hecho e hicieron del tema una de las banderas de crítica al gobierno. A pesar de que las desapariciones y homicidios han crecido en los últimos años, y de que Guerrero es uno de los estados con las tasas más elevadas, el alto número de víctimas y que éstas fueran estudiantes generó un escándalo en la opinión pública nacional e internacional. ${ }^{26}$ The New York Times, por ejemplo, reportó la noticia con el siguiente encabezado: "43 estudiantes desaparecidos, una fosa común y un solo sospechoso: la policía de México". ${ }^{27}$ The Washington Post se refirió a este hecho así: "Secuestro masivo de estudiantes en Iguala, México, provoca indignación y protestas". ${ }^{28}$ El Tiempo, de Bogotá, reportó la noticia de la siguiente manera: "Sicarios y policías envueltos en atroz crimen en México". ${ }^{29}$ Incluso el diario Le Monde expresó que las revelaciones sobre los estudiantes desaparecidos comen-

26 "Así informa la prensa internacional de lo que ocurre en Guerrero”, Aristegui Noticias, 7 de octubre de 2014, https:/ / aristeguinoticias.com/ 0710/mexico/asi-informa-la-prensa-internacional-lo-que-ocurre-en-guerrero/ (consulta del 17 de diciembre de 2018).

27 Randall C. Archibold, " 43 missing students, a mass grave and a suspect: Mexico’s police”, The New York Times, 6 de octubre de 2014, https:/ / www.nytimes.com/2014/10/07/world/americas/43-missing-students-amass-grave-and-a-suspect-mexicos-police-.html? ref=todayspaper\&_r=0 (consulta del 17 de diciembre de 2018).

${ }^{28}$ Nick Miroff, "Mass kidnapping of students in Iguala, Mexico, brings outrage and protests", The Washington Post, 11 de octubre de 2014, https:/ / www.washingtonpost.com/news/worldviews/wp/2014/10/11/in-mexico-mass-kidnapping-and-slaying-of-students-in-iguala-brings-outrage-andprotests-against-gangs-and-government/? noredirect=on\&utm_term=. cc7c764d795c (consulta del 18 de diciembre de 2018).

29 Alejandra Nóguez, "Sicarios y policías envueltos en atroz crimen en México”, El Tiempo, 6 de octubre de 2014, https://m.eltiempo.com/ archivo/documento/CMS-14649666 (consulta del 17 de diciembre de 2018). 
zaban a provocar una "crisis en seguridad". ${ }^{30}$ La presión nacional e internacional fue tan fuerte en este caso, que el gobierno de Peña Nieto suscribió a unos días del hecho un acuerdo de asesoría técnica con la Comisión Interamericana de Derechos Humanos (CIDH) de la oEA para la creación de un grupo de investigación independiente sobre los acontecimientos de Iguala. Como resultado de este acuerdo se creó el Grupo Interdisciplinario de Expertos Independientes (GIEI), formado por expertos legales y especialistas en derechos humanos de Chile, Colombia, Guatemala y España. Un año después, en septiembre de 2015, el GIEI presentó un reporte de su investigación, en el cual se refutaba la conclusión de la PGR de que los estudiantes habían sido incinerados en el basurero municipal de Cocula y se señalaba que la investigación oficial no había tomado en cuenta la presencia de un quinto autobús en el que viajaban los normalistas. ${ }^{31} \mathrm{El}$ procurador general de la República, Jesús Murillo Karam, había afirmado en enero de 2015 que los normalistas habían sido asesinados por el grupo criminal Guerreros Unidos, al confundirlos con miembros de un grupo rival, Los Rojos, y que habían sido quemados en el basurero de Cocula, una población aledaña a Iguala. ${ }^{32}$ Evidentemente, el informe del GIEI afectó seriamente la credibilidad internacional del gobierno de Peña Nieto. A raíz de este reporte, Amnistía Internacional declaró que éste había dejado al descubierto "la absoluta in-

${ }^{30}$ Fréderic Saliba, "Au Mexique l'armée investit Iguala où sont disparu les 43 étudiants", 7 de octubre de 2014. https:/ /www.lemonde.fr/ameriques/article/2014/10/07/mexique-l-etat-prend-le-controle-de-la-vi lle-ou-les-43-etudiants-ont-disparu_4501505_3222.html (consulta del 17 de diciembre de 2018).

${ }^{31}$ Grupo Interdisciplinario de Expertos Independientes, "Informe Ayotzinapa", septiembre de 2015. http://8d5306c18b850ea7e0ac-65b9b7a2fa68b3c92f951010bb26a4de.r54.cf2.rackcdn.com/pdf/Informe\% 20 ayotzi.pdf (consulta del 17 de diciembre de 2018).

32 Gustavo Castillo García, "Guerreros Unidos asesinó a los 43 normalistas: Murillo", La Jornada, 28 de enero de 2015, https://www.jornada. unam.mx/2015/01/28/politica/002n1pol\# (consulta del 17 de diciembre de 2018). 
competencia y falta de voluntad del gobierno para encontrar y castigar a los responsables". ${ }^{33}$ Por su parte, la organización Human Rights Watch señaló que "a pesar de que el mundo tenía los ojos puestos en México y de los enormes recursos asignados, las autoridades demostraron ser incapaces o no estar dispuestas a llevar a cabo una investigación seria”.34 Paralelamente a la labor del GIEI, las familias de los normalistas desparecidos, junto con el Centro de Derechos Humanos Miguel Agustín ProJuárez, el Centro de Derechos Humanos de la Montaña Tlachinollan de Guerrero, el Centro de Derechos Humanos José María Morelos y Pavón de Guerrero y los Servicios y Asesoría para la Paz, solicitaron, el 4 de octubre de 2014, al Equipo Argentino de Antropología Forense (EAAF) que realizara una investigación sobre los hecho de Iguala. El EAAF emitió el 8 de febrero de 2016 un reporte en el cual también refutaba la hipótesis de la PGR respecto a la incineración de los cuerpos de los estudiantes en el basurero de Cocula. ${ }^{35}$

Por otro lado, después del primer reporte del GIEI, la CIDH decidió prorrogar el mandato de dicho grupo hasta el 30 abril de 2016. Poco antes de esa fecha, el 24 de abril, el GIEI emitió un segundo reporte, en el cual se afirmaba que varios de los inculpados en la desaparición de los normalistas habían sido torturados y que elementos de la PGR realizaron diligencias en el basurero de Cocula y sus inmediaciones que no fueron reportadas. ${ }^{36} \mathrm{Al}$ término del mandato del GIEI el gobierno mexicano decidió no prorrogar el acuerdo con la CIDH para

${ }^{33}$ Juan Paullier, "México: Así reaccionó el gobierno al informe sobre los estudiantes de Ayotzinapa", BBC Mundo, 6 de septiembre de 2015, https://www.bbc.com/mundo/noticias/2015/09/150906_mexico_estudiantes_informe_ayotzinapa_giei_reaccion_gobierno_jp (consulta del 17 de diciembre de 2018).

${ }^{34}$ Loc. cit.

${ }^{35}$ Equipo Argentino de Antropología Forense, "Resumen ejecutivo", 8 de febrero de 2016, https://www.eaaf.org/files/informe_resumen_ejecutivo_08-02-2016.pdf (consulta del 17 de diciembre de 2018).

${ }^{36}$ Grupo Interdisciplinario de Expertos Independientes, "Informe Ayotzinapa II", https://www.oas.org/es/cidh/actividades/giei/giei-in formeayotzinapa2.pdf (consulta del 18 de diciembre de 2018). 
la permanencia de este grupo. Sin embargo, para entonces, era claro que el tema de la seguridad ocupaba ya un lugar prioritario en la agenda de la política exterior mexicana. Y esa situación perduró durante el resto del sexenio. De hecho, la entonces candidata a la presidencia de Estados Unidos, Hillary Clinton, declaró el 19 de abril de 2016, al referirse a la desaparición de los normalistas: "Es algo indignante. Si yo estuviese en el gobierno mexicano, no descansaría hasta descubrir lo que pasó con esos 42 ( sic) jóvenes. Su secuestro fue una terrible violación de la ley". ${ }^{37}$

En los años siguientes, el tema de Ayotzinapa siguió afectando la imagen internacional del gobierno mexicano, sin que las respuestas de éste fueran efectivas. Incluso, el 15 de marzo de 2018, la Organización de las Naciones Unidas (ONU) emitió un informe en el cual se concluía que había "fuertes elementos de convicción para considerar que al menos 34 personas detenidas durante la primera etapa de la investigación sobre la desaparición de 43 estudiantes de Ayotzinapa en 2014 fueron torturadas y muchas de ellas detenidas arbitrariamente. Estas serias violaciones fueron a su vez inadecuadamente investigadas y encubiertas". ${ }^{38}$ Llama la atención la respuesta del gobierno mexicano a este informe de la onU, la cual recuerda las décadas de los años setenta y ochenta, en

37 "Clinton: Si estuviese en el gobierno mexicano no descansaría hasta saber qué pasó con los normalistas”, La Jornada, 20 de abril de 2016, https:/ / www.jornada.com.mx/2016/04/20/politica/005n2pol\# (consulta del 18 de diciembre de 2018).

38 "Informe Ayotzinapa, México: la Oficina de Derechos Humanos de la onu respalda las conclusiones de su informe”, 9 de mayo de 2018, http:/ / hchr.org.mx/index.php?option=com_k2\&view=item\&cid=1114:inf orme-ayotzinapa-mexico-la-oficina-de-derechos-humanos-de-la-onu-res palda-las-conclusiones-de-su-informe\&Itemid=265 (consulta del 17 de diciembre de 2018); Oficina en México del Alto Comisionado de las Naciones Unidas para los Derechos Humanos, "Doble injusticia: Informe sobre violaciones de derechos humanos en la investigación del caso Ayotzinapa", marzo de 2018, http://hchr.org.mx/images/doc_pub/20180315_Doble Injusticia_InformeONUDHInvestigacionAyotzinapa.pdf (consulta del 17 de diciembre de 2018). 
las cuales se reivindicaba la soberanía para evitar críticas en temas espinosos, como los de la democracia y los derechos humanos. En su respuesta, el gobierno señaló que el informe carecía "de rigor metodológico y sustento jurídico para arribar a la conclusión de que se está cometiendo una «doble injusticia» como su mismo título lo describe" y que dicho organismo estaba asumiendo funciones que "sólo corresponden a un juzgador”, lo cual desvirtuaba la función que se acordó realizaría en el país. ${ }^{39}$

Además del caso Ayotzinapa, hubo otros sucesos relacionados con el combate a la delincuencia organizada que también repercutieron negativamente en la imagen externa de México. Uno de ellos fue la muerte de 22 supuestos criminales por parte del Ejército Mexicano en el poblado de Tlatlaya en el Estado de México el 30 de junio de 2014. La versión oficial decía que esas personas habrían muerto en un enfrentamiento con las fuerzas federales. Sin embargo, en septiembre del mismo año, la agencia noticiosa Associated Press y la revista Esquire Latinoamérica publicaron sendos reportajes, en los cuales una mujer -supuestamente una de las secuestradas que fueron rescatadas por el Ejército-, afirmaba que 21 de los sospechosos habían sido ejecutados por los militares a pesar de haberse rendido y salir con las manos en la nuca. Como resultado de esta información, la Secretaría de la Defensa inició una investigación, tras la cual 17 militares fueron procesados, de los cuales seis fueron liberados posteriormente. Sólo uno purgó una pena de un año. En agosto de 2018, un juez ordenó reabrir el caso por deficiencias en la investigación. ${ }^{40}$ Como ocurrió con los estudiantes

39 "Informe de la ONU sobre caso Ayotzinapa fue más allá de sus atribuciones: gobierno de México", Aristegui Noticias,7 de mayo de 2018, https:/ / aristeguinoticias.com/0705/mexico/informe-de-la-onu-sobre-caso-ayotzi napa-fue-mas-alla-de-sus-atribuciones-gobierno-de-mexico/ (consulta del 17 de diciembre de 2018).

${ }^{40}$ Fernando Camacho y Emir Olivares, "Ordena juez a la PGR reabrir el caso Tlatlaya", La Jornada, 12 de agosto de 2018, https://www.jornada.com. $\mathrm{mx} / 2018 / 08 / 12 /$ politica/002n1 pol (consulta del 17 de diciembre de 2018). 
de Ayotzinapa, la matanza de Tlatlaya también ocasionó fuertes críticas de la prensa internacional y las organizaciones no gubernamentales de derechos humanos, como Human Rights Watch, cuyo director de la División para las Américas, José Miguel Vivanco, la calificó como "la peor masacre de civiles por parte de militares de este sexenio". ${ }^{41}$ Asimismo, el Centro de Derechos Humanos Miguel Agustín Pro Juárez publicó un informe a mediados de 2015, en el cual se señala que los militares tenían la orden de abatir a los delincuentes. ${ }^{42}$

De esta forma, a finales del sexenio de Peña Nieto, la agenda internacional se había ya seguritizado, a pesar de los esfuerzos del gobierno por evitarlo. Pero ello no se debió sólo al caso Ayotzinapa o al caso Tlatlaya. Lo cierto es que la estrategia del gobierno mexicano para combatir la inseguridad resultó un fracaso. No sólo la tasa de homicidios comenzó a subir de manera constante a partir de 2014 (cuando fue de 17 por cada 100000 habitantes) hasta llegar a 25 por cada 100000 habitantes en 2018, frente a una tasa de 24 por cada 100000 habitantes en 2011, en el punto más alto de la violencia con Calderón, sino que la tasa de prevalencia delictiva en el sexenio aumentó en $8.81 \%$, de 27337 por cada 100000 habitantes, en 2012, a 29746 por cada 100000 habitantes, en $2017 .{ }^{43}$

41 "Tlatlaya, primera gran masacre del gobierno de EPN, según HRw, se vuelve tema mundial", Sin Embargo, 19 de septiembre de 2014, https:/ / www.sinembargo.mx/19-09-2014/1121286 (consulta del 17 de diciembre de 2018).

42 Ana Langner, "La orden a los militares fue abatir a los delincuentes", El Economista, 2 de julio de 2015, https:/ / www.eleconomista.com.mx/ politica/La-orden-a-los-militares-fue-abatir-a-los-delincuentes-20150702-0085.html (consulta del 17 de diciembre de 2018).

43 "INEGI. Encuesta Nacional de Victimización y Percepción sobre Seguridad Pública (ENVIPE) 2018”, Boletín de prensa núm. 425/18, 25 de septiembre de 2018, http://www.beta.inegi.org.mx/contenidos/saladeprensa/boletines/2018/EstSegPub/envipe2018_09.pdf (consulta del 17 de diciembre de 2018). 


\section{LA RELACIÓN CON ESTADOS UNIDOS: LA SEGURIDAD COMO HERRAMIENTA DE NEGOCIACIÓN}

Como ya apuntamos, el esfuerzo del gobierno de Peña Nieto por deseguritizar la política exterior se reflejó también en la relación con Estados Unidos. Aunque no se canceló la Iniciativa Mérida, dejó de ser un referente de la relación bilateral. A pesar de ello, la seguridad continuó siendo un tema central en esta relación. La colaboración entre las agencias de inteligencia de ambos países continuó con regularidad durante la administración de Peña Nieto. Incluso, en una reunión en julio de 2013 entre el secretario de Gobernación, Miguel Ángel Osorio Chong, y la secretaria de Seguridad Interior de Estados Unidos, Janet Napolitano, esta última señaló "el énfasis en el intercambio de inteligencia, haciendo evaluación de los riesgos que ambos países enfrentan [...] esas cosas creo que están continuando y que son más fuertes de lo que han sido". ${ }^{44}$ La colaboración en materia de inteligencia fue clave para la captura del líder de los Zetas, Miguel Ángel Treviño Morales, en $2013^{45}$ y la recaptura del narcotraficante Joaquín “Chapo” Guzmán, en 2014. ${ }^{46}$

Por otro lado, a medida que la crisis de seguridad se hizo patente en el país, el gobierno de Peña Nieto aumentó la adquisición de armas a Estados Unidos. Para junio de 2015, el gobierno mexicano había gastado alrededor de 3500 millones de dólares en armamento, una cifra "sin precedentes", de

44 Associated Press, "DHs secretary discusses cooperation with Mexican domestic security chief at border meeting”, Fox News, 23 de julio de 2013, https://www.foxnews.com/world/dhs-secretary-discusses-coopera tion-with-mexican-domestic-security-chief-at-border-meeting (consulta del 17 de diciembre de 2018).

${ }^{45}$ Loc. cit.

${ }^{46}$ Catherine E. Shoichet y Evan Pérez, "Cómo lograron capturar a «el Chapo» Guzmán, el narcotraficante más buscado del mundo”, CNN Español, 24 de febrero de 2014, https://cnnespanol.cnn.com/2014/02/24/ como-lograron-capturar-a-el-chapo-guzman-el-narcotraficante-mas-busca do-del-mundo/ (consulta del 17 de diciembre de 2018). 
acuerdo con The Washington Post. Estas compras incluían "al menos dos docenas de helicópteros Blackhawk UH-60 -de la fuerza aérea y la marina estadounidenses- y unos 2200 vehículos blindados Humvee”. Llama la atención la declaración de un oficial estadounidense ante estas compras, que confirma que el esfuerzo por deseguritizar la política exterior fue fallido: "Sabíamos que el presidente Peña Nieto quería enfocarse en otras cosas diferentes a la seguridad [...] Después se dio cuenta de que tenía que poner atención al tema de la seguridad". ${ }^{47}$ Estas compras de armamento para reforzar las capacidades del gobierno en el combate al crimen organizado continuaron hasta el fin del sexenio. ${ }^{48}$ Otro hecho que refleja la creciente importancia del tema de la seguridad y el interés del gobierno de Peña Nieto en colaborar con Estados Unidos en este rubro fue la autorización, en 2015, de "la portación temporal de armas a los servidores públicos extranjeros de migración o aduanas, debidamente acreditados ante el gobierno federal, que participen en la revisión migratoria en los puntos de tránsito internacionales o el despacho conjunto de mercancías en las aduanas nacionales". ${ }^{49}$

Otra razón para que la seguridad se volviera un tema central en la relación con Estados Unidos, además de la crisis de inseguridad que se hizo evidente al poco tiempo de iniciado el

47 “Peña Nieto ha comprado armas 'sin precedentes' a Eu: más de 3 mil mdd”, Animal Político, 16 de junio de 2011, https://www.animalpoliti co.com/2015/06/pena-nieto-ha-comprado-armas-sin-precedentes-a-eumas-de-3-mil-mdd (consulta del 17 de diciembre de 2018). La nota aludida de The Washington Post es: Joshua Partlow, "What's behind Mexico's military buying binge?", The Washington Post, https://www.washingtonpost. com/news/worldviews/wp/2015/06/15/whats-behind-mexicos-militarybuying-binge/?utm_term=.30965fd57c64

48 "México comprará armamento militar a Estados Unidos por 98.4 millones de dólares", Animal Político, 6 de enero de 2018, https://www. animalpolitico.com/2018/01/mexico-eu-compra-armamento/ (consulta del 17 de diciembre de 2018).

${ }^{49}$ Decreto por el que se reforman y adicionan diversas disposiciones de la Ley Federal de Armas de Fuego y Explosivos, Diario Oficial de la Federación, 22 de mayo de 2015. 
sexenio, fue que, con la llegada al poder de Donald Trump, el gobierno de Peña Nieto se dio cuenta de que la principal herramienta de negociación que tenía frente a ese gobierno era precisamente ese tema. De hecho, ante las constantes críticas de Trump a los migrantes mexicanos y las amenazas de cancelar el Tratado de Libe Comercio de América del Norte, la cancillería mexicana le hizo saber al presidente y al Congreso de Estados Unidos que el Senado mexicano le había pedido suspender la cooperación bilateral en materia de seguridad y migración en tanto el mandatario de ese país no se condujera "con respeto" hacia México. ${ }^{50}$

Desde esta perspectiva, el manejo de la migración de centroamericanos que buscan llegar a Estados Unidos -tema que el gobierno de Trump seguritizó desde su campaña presidencial- fue también una herramienta de negociación frente a la Casa Blanca. Así, desde la década pasada, el gobierno mexicano ha colaborado con Washington para controlar el flujo migratorio de Centroamérica hacia el país del norte. Esta política la continuó Peña Nieto. De 2015 a 2018, México deportó a 436125 guatemaltecos, salvadoreños y hondureños, cifra considerablemente mayor que los 293813 que deportó el gobierno de Estados Unidos en el mismo periodo. ${ }^{51}$ Incluso, según The Washington Post, el gobierno de Trump habría alcanzado un acuerdo con el gobierno entrante de López Obrador para que los migrantes centroamericanos en México permanecieran en México hasta que sus peticiones de

${ }^{50}$ Iván E. Saldaña, "SRE hace saber a Trump que Senado pidió suspender cooperación bilateral”, Excélsior, 24 de abril de 2018, https: / / www. excelsior.com.mx/nacional/sre-hace-saber-a-trump-que-senado-pidiosuspender-cooperacion-bilateral/1234543 (consulta del 17 de diciembre de 2018).

51 José Meléndez, "México rebasa a Eu en expulsión de migrantes", $E l$ Universal, 21 de octubre de 2018, https://www.eluniversal.com.mx/mun do/mexico-rebasa-eu-en-expulsion-de-migrantes?fbclid=IwAR3BNIxx5m KsdC0NFnQrmCwTNHpACvULuIattnRVhrBwRqXmcn6LXR3x5Ms (consulta del 17 de diciembre de 2018). 
asilo fueran procesadas en la cortes de Estados Unidos. ${ }^{52} \mathrm{Di}$ cho acuerdo habría sido negado posteriormente por la secretaria de Gobernación, Olga Sánchez Cordero.

A finales del sexenio de Peña Nieto se presentó otra crisis en la frontera con Estados Unidos, como resultado de la llegada masiva de migrantes centroamericanos a la ciudad de Tijuana, que ingresaron a México en varias caravanas a través de la frontera sur. La presencia de los migrantes en esa ciudad fronteriza provocó fuertes reacciones xenofóbicas de parte de la población tijuanense y una saturación de los servicios básicos que no pudo ser resuelta satisfactoriamente ni por el gobierno local ni por el gobierno federal. Si bien la entrada de migrantes de Centroamérica no es nueva, el hecho de que se haya dado masivamente es inédito y evidenció la falta de una estrategia del gobierno mexicano para lidiar con un fenómeno de esas características.

\section{Conclusiones}

Como ha podido apreciarse a lo largo de este artículo, desde el inicio de su gobierno, el presidente Peña Nieto decidió deseguritizar la política exterior por varias razones. Primero, este proceso era la cara externa de una política interna en el mismo sentido que buscaba distanciarse del gobierno de su antecesor, cuya estrategia de seguridad fue considerada como la causante del incremento de la violencia en el país por el partido de Peña Nieto -y por la oposición de izquierda-. Segundo, la deseguritización de la política exterior era un requisito fundamental para presentar a México como una potencia económica con capacidad de liderazgo internacional. Sin

${ }^{52}$ Joshua Partlow y Nick Miroff, "Deal with Mexico paves the way for asylum overhaul at us border", The Washington Post, 24 de noviembre de 2018, https://www.washingtonpost.com/world/national-security/deal-wi th-mexico-paves-way-for-asylum-overhaul-at-us-border/2018/11/24/87b 9570a-ef74-11e8-9236-bb94154151d2_story.html?noredirect=on\&utm_ term=.69a9826d2cfb (consulta del 17 de diciembre de 2018). 
embargo, al final, el tema de la seguridad se coló en la política exterior de Peña Nieto como un invitado incómodo. Su inclusión en la agenda exterior fue resultado de dos circunstancias que no se tenían previstas al inicio del sexenio: un incremento de la inseguridad en México y la llegada de Donald Trump a la presidencia de Estados Unidos. El incremento de la inseguridad, medido con las cifras de homicidios y los índices de criminalidad, fue probablemente resultado de la falta de continuidad en las políticas de seguridad disenadas por el gobierno anterior -que ya habían incidido en una baja en la tasa de homicidios a inicios del sexenio de Peña Nieto-, así como de crisis muy concretas que afectaron seriamente la imagen del gobierno: la aparición de los grupos de autodefensa en Michoacán y la intervención del gobierno federal en esa entidad, la desaparición de los normalistas de Ayotzinapa y la muerte de civiles a manos del Ejército en Tlatlaya. En ese sentido, el hecho de que se buscara deliberadamente disminuir el tema de la seguridad en la agenda interna y externa del gobierno hizo que las respuestas que éste tuvo ante las crisis en este ámbito fueran desarticuladas e ineficientes. Al no figurar en la agenda inicial del gobierno como un tema prioritario, no había una estrategia para lidiar con los efectos de las crisis que se generaron y que provocaron un serio deterioro de la imagen internacional del país. Ello fue evidente en los casos de Ayotzinapa y Tlatlaya, y con la llegada masiva de migrantes a Tijuana a finales del sexenio. Al mismo tiempo, aunque el tema se reveló como una fuerte carta de negociación frente a Estados Unidos, el que no formara parte de la agenda internacional inicial le restó efectividad. Desde esta perspectiva, todo indica que el próximo gobierno está más consciente de la relevancia interna y externa de la seguridad y que ésta estará presente en ambas agendas. Lamentablemente, dada la profundidad de la crisis en el rubro, es difícil que el tema pase en forma natural a un segundo plano. En todo caso, el sexenio de Peña Nieto muestra que, aunque los gobiernos son actores fundamentales en el proceso de seguritización (o deseguritización) 
de algunos asuntos, al final, éste no es sólo un acto retórico, sino que debe tener una correspondencia con la realidad.

\section{Bibliografía}

Archibold, Randall C., "A Quandary for Mexico as vigilantes rise”, The New York Times, 15 de enero de 2014, https:/ / www. nytimes.com/2014/01/16/world/americas/a-quandary-formexico-as-vigilantes-rise.html (consulta del 7 de diciembre de 2018).

ArChibold, Randall C., "43 missing students, a mass grave and a suspect: Mexico's police”, The New York Times, 6 de octubre de 2014, https://www.nytimes.com/2014/10/07/world/americas /43-missing-students-a-mass-grave-and-a-suspect-mexicos-poli ce-html?ref=todayspaper\&_r=0 (consulta del 17 de diciembre de 2018).

"Así informa la prensa internacional de lo que ocurre en Guerrero", Aristegui Noticias, 7 de octubre de 2014, https://aristegui noticias.com/0710/mexico/asi-informa-la-prensa-internacion al-lo-que-ocurre-en-guerrero/ (consulta del 17 de diciembre de 2018).

Beltrán, Ulises y Alejandro Cruz, "Crece percepción de violencia”, Excélsior, 11 de noviembre de 2013, http://www.bgc.com. $\mathrm{mx} /$ archivos/excelsior/2013/noviembre/EX111113.pdf (consulta del 17 de diciembre de 2018.

Bewley-Taylor, David R., International Drug Control: Consensus fractured, Cambridge, University Press, 2012.

Buzan, Barry, Ole Włver y Jaap de Wilde, Security: A New Framework for Analysis, Boulder, Lynne Rienner Publishers, 1998.

CAMACHO, Eduardo, "SRE: política exterior apoyará al comercio", $E l$ Universal, 17 de abril de 2013, http:/ / www.eluniversal.com.mx/ finanzas/101867.html (consulta del 17 de diciembre de 2018).

Camacho, Fernando y Emir Olivares, "Ordena juez a la PGR reabrir el caso Tlatlaya”, La Jornada, 12 de agosto de 2018, https:/ / www.jornada.com.mx/2018/08/12/politica/002n1pol (consulta del 7 de diciembre de 2018). 
CAstañeda, Jorge G., "Salinas' international relations gamble", Journal of International Affairs, núm. 2 (1990), pp. 407-422.

Castillo García, Gustavo, "Guerreros Unidos asesinó a los 43 normalistas: Murillo", La Jornada, 28 de enero de 2015, https:/ / www.jornada.unam.mx/2015/01/28/politica/002n1pol\# 1 (consulta del 17 de diciembre de 2018).

Снават, Jorge, "Mexico's foreign policy in 1990: Electoral Sovereignty and integration with the United States", Journal of Interamerican Studies and World Affairs, núm. 4 (1991).

"Clinton: Si estuviese en el gobierno mexicano no descansaría hasta saber qué pasó con los normalistas", La Jornada, 20 de abril de 2016, https://www.jornada.com.mx/2016/04/20/politica/ 005n2pol\# (consulta del 18 de diciembre de 2018).

Decreto por el que se reforman y adicionan diversas disposiciones de la Ley Federal de Armas de Fuego y Explosivos, Diario Oficial de la Federación, 22 de mayo de 2015.

"DHs secretary discusses cooperation with Mexican domestic security chief at border meeting", Fox News, 23 de julio de 2013, https://www.foxnews.com/world/dhs-secretary-discusses-cooperation-with-mexican-domestic-security-chief-at-border-meeting (consulta del 17 de diciembre de 2018).

Equipo Argentino de Antropología Forense, "Resumen ejecutivo", 8 de febrero de 2016, https://www.eaaf.org/files/informe_resumen_ejecutivo_08-02-2016.pdf 17 (consulta del 17 de diciembre de 2018).

"Gobernación, única ventanilla para tratar temas de seguridad con Estados Unidos", Animal Político, 30 de abril de 2013, https:/ / www.animalpolitico.com/2013/04/gobernacion-la-unica-ventanilla-para-tratar-tema-de-seguridad-con-eu/ (consulta del 17 de diciembre de 2018).

Gómez, Natalia y Ariadna García, "México y eu forman grupo de alto nivel en seguridad", El Universal, 12 de octubre de 2016, http:/ /www.eluniversal.com.mx/articulo/nacion/seguridad/ 2016/10/12/mexico-y-eu-forman-grupo-de-alto-nivel-en-segu ridad (consulta del 17 de diciembre de 2018.

Grupo Interdisciplinario de Expertos Independientes, "Informe Ayotzinapa”, septiembre de 2015, http:/ /8d5306c18b850 ea7e 
0ac-65b9b7a2fa68b3c92f951010bb26a4de.r54.cf2.rackcdn. com/pdf/Informe\%20ayotzi.pdf (consulta del 17 de diciembre de 2018).

Grupo Interdisciplinario de Expertos Independientes, "Informe Ayotzinapa II", https:/ /www.oas.org/es/cidh/actividades/giei/ giei-informeayotzinapa2.pdf (consulta del 17 de diciembre de 2018).

Guerrero, Claudia, "Plantea Peña Nieto crear Gendarmería”, Reforma, 10 de mayo de 2012.

Guzmán, Susana, "Centro de fusión de inteligencia coordinará acciones para fortalecer seguridad: Segob”, El Financiero, 2 de noviembre de 2016, http://www.elfinanciero.com.mx/nacional/centro-de-fusion-de-inteligencia-coordinara-acciones-pa ra-fortalecer-seguridad-segob (consulta del 17 de diciembre de 2018).

Hernández, Erika, "Peña Nieto asegura que afianzó lazos con Asia", Terra Noticias, 8 de octubre de 2012, http:/ / noticias.terra.com.mx/mexico/politica/pena-nieto-asegura-que-mexicoafianzo-lazos-con-asia,66ab808345891410VgnVCM5000009ccc eb0aRCRD.html (consulta del 14 de octubre de 2014).

"Informe Ayotzinapa, México: la Oficina de Derechos Humanos de la onu respalda las conclusiones de su informe", 9 de mayo de 2018, http://hchr.org.mx/index.php?option=com_k2\&vie $\mathrm{w}=$ item\&id=1114:informe-ayotzinapa-mexico-la-oficina-de-derechos-humanos-de-la-onu-respalda-las-conclusiones-de-suinforme\&Itemid=265 (consulta del 17 de diciembre de 2018). "Informe de la onu sobre caso Ayotzinapa fue más allá de sus atribuciones: gobierno de México", Aristegui Noticias, 7 de mayo de 2018, https:/ /aristeguinoticias.com/0705/mexico/informe-dela-onu-sobre-caso-ayotzinapa-fue-mas-alla-de-sus-atribucionesgobierno-de-mexico/ (consulta del 17 de diciembre de 2018).

Instituto Nacional de Estadística y Geografía, INEGI, Encuesta Nacional de Victimización y Percepción sobre Seguridad Pública (ENVIPE) 2018", 25 de septiembre de 2018, http:/ / www.beta.inegi. org.mx/contenidos/saladeprensa/boletines/2018/EstSegPub/ envipe2018_09.pdf (consulta del 17 de diciembre de 2018). 
LANGNER, Ana, "La orden a los militares fue abatir a los delincuentes", El Economista, 2 de julio de 2015, https://www.eleconomista.com.mx/politica/La-orden-a-los-militares-fue-aba tir-a-los-delincuentes-20150702-0085.html (consulta del 17 de diciembre de 2018).

Meléndez, José, "México rebasa a eu en expulsión de migrantes", El Universal, 21 de octubre de 2018, https://www.eluniversal. com.mx/mundo/mexico-rebasa-eu-en-expulsion-de-migrante s?fbclid=IwAR3BNIxx5mKsdC0NFnQrmCwTNHpACvULuIat tnRVhrBwRqXmcn6LXR3x5Ms (consulta del 17 de diciembre de 2018).

"México comprará armamento militar a Estados Unidos por 98.4 millones de dólares", Animal Político, 6 de enero de 2018, https:/ / www.animalpolitico.com/2018/01/mexico-eu-compra-arma mento/ (consulta del 17 de diciembre de 2018).

"México se compromete a ser un actor con mayor responsabilidad global: Enrique Peña Nieto”, Presidencia de la República, 14 de febrero de 2013, http:/ /www.presidencia.gob.mx/articulosprensa/mexico-se-compromete-a-ser-un-actor-con-mayor-res ponsabilidad-global-enrique-pena-nieto/ (consulta del $14 \mathrm{de}$ octubre de 2014).

Miroff, Nick, "Mass kidnapping of students in Iguala, Mexico, brings outrage and protests", The Washington Post, 11 de octubre de 2014, https://www.washingtonpost.com/news/worldviews/wp/2014/10/11/in-mexico-mass-kidnapping-and-slayi ng-of-students-in-iguala-brings-outrage-and-protests-againstgangs-and-government/?utm_term=.fc25f06307d1 (consulta del 17 de diciembre de 2018).

Montalvo, Tania L.,"Un México en paz debe tener el apoyo de la diplomacia: canciller Meade", Expansión, 7 de enero de 2013, https:/ / expansion.mx/nacional/2013/01/07/un-mexico-enpaz-debe-tener-el-apoyo-de-la-diplomacia-canciller-meade 1 (consulta del 17 de diciembre de 2018).

Naciones Unidas, "Discurso del presidente Enrique Peña Nieto en la ungass 2016", Boletín, Comunicado núm. 16/083,19 de abril de 2016, http://www.cinu.mx/comunicados/2016/04/ 
el-problema-mundial-de-las-dro/ (consulta del 17 de diciembre de 2018).

"Naranjo propone plan para reducir violencia en México en 100 días", El Espectador, 7 de julio, https:/ / www.elespectador.com/ noticias/elmundo/naranjo-propone-plan-reducir-violenciamexico-100-dias-articulo-357979 (consulta del 17 de diciembre de 2018).

Nóguez, Alejandra, "Sicarios y policías envueltos en atroz crimen en México”, El Tiempo, 6 de octubre de 2014, https://m.eltiem po.com/archivo/documento/CMS-14649666 (consulta del 17 de diciembre de 2018).

Oficina en México del Alto Comisionado de las Naciones Unidas para los Derechos Humanos, "Doble injusticia: Informe sobre violaciones de derechos humanos en la investigación del caso Ayotzinapa”, http://hchr.org.mx/images/doc_pub/20180315 _DobleInjusticia_InformeONUDHInvestigacionAyotzinapa. pdf (consulta del 17 de diciembre de 2018).

Partlow, Joshua, "What's behind Mexico's military buying binge?", The Washington Post, 15 de junio de 2015 https://www. washingtonpost.com/news/worldviews/wp/2015/06/15/wha ts-behind-mexicos-mi litary-buying-binge $/$ ?utm_term $=.30965 \mathrm{f}$ d57c64

Partlow, Joshua y Nick Miroff, "Deal with Mexico paves the way for asylum overhaul at us border", The Washington Post, 24 de noviembre de 2018, https://www.washingtonpost.com/world/ national-security/deal-with-mexico-paves-way-for-asylum-overhaul-at-us-border/2018/11/24/87b9570a-ef74-11e8-9236-bb 94154151 d2_story.html?noredirect $=$ on\&utm_term $=.69$ a 9826 d2cfb (consulta del 17 de diciembre de 2018).

Paullier, Jean, "México: Así reaccionó el gobierno al informe sobre los estudiantes de Ayotzinapa", BBC Mundo, 6 de septiembre de 2015, https:/ / www.bbc.com/mundo/noticias/2015/09/ 150906_mexico_estudiantes_informe_ayotzinapa_giei_reac cion_gobierno_jp (consulta del 17 de diciembre de 2018).

"Peña Nieto presenta propuestas de gobierno en Davos", El Economista, 27 de enero de 2012, https://www.eleconomista.com. $\mathrm{mx} /$ politica/Pena-Nieto-presenta-propuestas-de-gobierno-en- 
Davos-20120127-0012.html (consulta del 17 de diciembre de 2018).

Peña Nieto, Enrique, México, la gran esperanza, México, Grijalbo, 2012.

Peña Nieto, Enrique, "Mexico's moment”, The Economist, 21 de noviembre de 2012, https://www.economist.com/news/215663 14-enrique-pe \% C3\% B1a-nieto-mexicos-newly-elected-presi dent-sets-out-his-priorities-mexicos-moment (consulta del $17 \mathrm{de}$ diciembre de 2018).

"Peña Nieto ha comprado armas 'sin precedentes' a Eu: más de 3 mil mdd”, Animal Político, 16 de junio de 2011, https://www. animalpolitico.com/2015/06/pena-nieto-ha-comprado-armas-sin-precedentes-a-eu-mas-de-3-mil-mdd (consulta del 17 de diciembre de 2018).

"Peña Nieto prepara paquete de reformas estructurales", El Economista, 2 de julio de 2012, https://www.eleconomista.com.mx/ economia/Pena-Nieto-prepara-paquete-de-reformas-estructura les-20120702-0102.html (consulta del 17 de diciembre de 2018).

"Peña propone reformular estrategia de seguridad", El Economista, 28 de abril de 2012, https:/ /www.eleconomista.com.mx/poli tica/Pena-propone-reformular-estrategia-en-seguridad-20 120428-0004.html (consulta del 17 de diciembre de 2018).

“¿Qué es el Programa Nacional para la Prevención social de la Violencia y el Delito?”, México, http:/ / mexicoevalua.org/prevencion/conoce-el-proyecto/que-es-el-programa-nacional-pa ra-la-prevencion-social-de-la-violencia-y-la-delincuencia-pronapred/ (consulta del 17 de diciembre de 2018).

Ramírez Meda, Kenia y Nalia María Rochin Aguilar, "La política exterior de México durante el sexenio de Enrique Peña Nieto", Journal of International Relations, núm. 8 (2017), pp. 51-66.

SALDAÑA, Iván E., "sRe hace saber a Trump que Senado pidió suspender cooperación bilateral”, Excélsior, 24 de abril de 2018, https://www.excelsior.com.mx/nacional/sre-hace-saber-atrump-que-senado-pidio-suspender-cooperacion-bilateral/1234543 (consulta del 17 de diciembre de 2018).

SAliba, Fréderic, "Au Mexique l'armée investit Iguala où sont disparu les 43 étudiants”, 7 de octubre de 2014, https://www.le 
monde.fr/ameriques/article/2014/10/07/mexique-l-etat-pre nd-le-controle-de-la-ville-ou-les-43-etudiants-ont-disparu_450 1505_3222.html (consulta del 17 de diciembre de 2018). "Segob publica decreto que crea la División de Gendarmería", $E l$ Financiero, 22 de agosto de 2014, http:/ / www.elfinanciero.com. $\mathrm{mx} /$ sociedad/segob-publica-decreto-que-crean-la-division-degendarmeria (consulta del 17 de diciembre de 2018).

Shoichet, Catherine E. y Evan Pérez, "Cómo lograron capturar a «el Chapo» Guzmán, el narcotraficante más buscado del mundo”, CNN Español, 24 de febrero de 2014, https://cnnespanol. cnn.com/2014/02/24/como-lograron-capturar-a-el-chapoguzman-el-narcotraficante-mas-buscado-del-mundo/ (consulta del 17 de diciembre de 2018).

"Tlatlaya, primera gran masacre del gobierno de EPN, según HRw, se vuelve tema mundial", Sin Embargo, 19 de septiembre de 2014, https:/ /www.sinembargo.mx/19-09-2014/1121286 (consulta del 17 de diciembre de 2018). 